\title{
Comunicação terapêutica na Enfermagem: dificuldades para o cuidar de idosos com câncer
}

Therapeutic communication in Nursing: difficulties for the care of elderly people with cancer

Comunicación terapéutica en Enfermería: dificultades para el cuidado de ancianos con cáncer

Aline Azevedo Peterson', Emília Campos de Carvalho'

' Universidade de São Paulo, Escola de Enfermagem de Ribeirão Preto. Ribeirão Preto-SP, Brasil.

Submissão: 2/11/2010 Revisão: 21/11/2011 Aprovação: 5/12/2011

\section{RESUMO}

O objetivo deste trabalho foi avaliar as dificuldades e suas respectivas causas, na percepção do enfermeiro, ao se prestar assistência ao paciente idoso, com patologia oncológica. O estudo, descritivo, utilizou a Técnica dos Incidentes Críticos para obtenção e análise de dados, respeitados os aspectos éticos. Os sujeitos foram enfermeiros, convidados, funcionários de Unidades de assistência oncológica às pessoas com idade superior a 60 anos e que atuam nas áreas de oncologia há mais de um ano. Foram citados pelos sujeitos 25 incidentes críticos, sendo nove sentimentos negativos, três positivos e 13 com ambos. Concluiu-se que os enfermeiros que relataram intervenções de caráter humano, demonstraram sentimentos positivos, reconhecendo a importância das respectivas ações de enfermagem para oferecer uma assistência humana.

Descritores: Enfermagem Geriátrica; Assistência de enfermagem; Cuidados paliativos; Oncologia; Comunicação.

\begin{abstract}
The objective of this study is to assess the difficulties and their causes, in the perception of nurses, to give aid to the old patient bearer oncologic pathology. The study, descriptive, used The Critical Incident Technique to the obtaining and analyzing of data, complied with the ethical. The subjects were nurses, guests, employees of units of cancer care to people aged over 60 years and working in oncology for more than a year. The subjects cited 25 critical incidents, while 9 situations generated negative feelings, 3 positives and 13 with double feelings. It was concluded that nurses who reported interventions of human character, showed positive feelings, recognizing how important their nursing actions were important to offer human assistance.
\end{abstract}

Key words: Nursing care; Geriatric Nursing; Palliative care; Oncology; Communication.

\section{RESUMEN}

El objetivo de este estudio fue avaluar los problemas e sus causas, la percepción de la enfermera en la prestación de asistencia a los pacientes de edad avanzada que sufren de neoplasias. El estudio, descriptivo, utilizó la Técnica del Incidente Crítico para la obtención y análisis de los datos, cumplido con la ética. Los sujetos fueran enfermeros, los clientes, los empleados de las unidades de atención del cáncer a las personas mayores de 60 años y trabajo en oncología desde hace más de un año. Fueran citados 25 incidentes críticos por los sujetos, y 9 posiciones han generado sentimientos negativos, tres positivos y 13 dobles. Se concluyó que las enfermeras que informaron de diversas intervenciones de carácter humano, mostró sentimientos positivos, reconociendo la importancia de las acciones de enfermería para proporcionar asistencia humana.

Palabras clave: Cuidado de enfermería; Enfermería Geriátrica; Cuidados paliativos; Oncología; Comunicación. 


\section{INTRODUÇÃO}

Na Enfermagem, para um cuidado humano e individualizado, é preciso utilizar mais do que conhecimentos científicos; é necessário estabelecer uma relação na qual o enfermeiro esteja sempre disposto a ouvir o paciente e a informar-lhe a respeito de seu tratamento. A comunicação permite transmitir informações claras e objetivas, para proporcionar maiores escolhas e resoluções, tornando-se mais uma forma de o paciente sanar suas dúvidas a respeito de sua doença, sendo indispensável para uma assistência de qualidade. Em vista disso, ela colabora para a promoção do cuidado emocional, que é a habilidade de perceber o imperceptível ${ }^{(1)}$, exigindo alto nível de sensibilidade para as manifestações verbais e não verbais do cliente que possam indicar ao enfermeiro suas necessidades individuais.

A comunicação em enfermagem, empregada de forma terapêutica, permite que o profissional procure ajudar os pacientes a se adaptarem melhor às situações, identificando e atendendo suas necessidades de saúde, além de transmitir-lhe confiança, a fim de que se sintam satisfeitos e seguros, diminuindo o medo e a ansiedade, permitindo participar do seu tratamento. Além disso, para alguns autores, a comunicação terapêutica é fundamental para um cuidado humanizado e na demonstração de respeito por parte do enfermeiro ${ }^{(2)}$.

Portanto, o enfermeiro deve desenvolver uma comunicação adequada e efetiva, utilizando-se de procedimentos técnicos, escuta e atenção adequada ${ }^{(3)}$. É necessário que haja diálogo constante entre ambos, cultivando a confiança, o respeito e a empatia, para que, contribua no processo de restabelecimento do paciente.

Entretanto, devido ao medo da morte, ansiedade do enfermeiro sobre a habilidade do cliente de enfrentar a doença, falta de tempo ou falta de treinamento de como interagir com estes clientes, há dificuldades de comunicação entre enfermeiro e pacientes em estado terminal(4). O cuidar de pacientes nesta condição confronta os enfermeiros com seus próprios medos relacionados com a possibilidade de sofrer e morrer. Os temores e ansiedades não reconhecidos podem interferir no cuidado de enfermagem oferecido aos pacientes ${ }^{(5)}$.

A falta de vontade de se comunicar ou de interagir com os doentes é interpretada por estes como um não cuidado, além do que uma atitude distante pode gerar sentimentos de medo e abandono. Soma-se ainda, após saberem do diagnóstico de câncer, os pacientes esperam receber apoio do profissional de saúde e, ao manifestarem suas ansiedades, preocupações e dúvidas, receberem informação do enfermeiro ${ }^{(6)}$.

Tal quadro é preocupante, pois ao enfermeiro também compete fornecer informações ou esclarecer o doente de câncer sobre questões técnicas, bem como dar apoio emocional e informacional adequado e compatível às necessidades destes doentes, desenvolver habilidades de enfrentamento e lutar pelo seu restabelecimento ${ }^{(7)}$.

A complexidade do tratamento com câncer requer habilidades tanto técnico-científica como de relações interpessoais. O conhecimento somado com afetividade, comunicação, sinceridade e empatia, formam elementos construtivos para o cuidado, os quais estarão influenciando o desenvolvimento da assistência prestada ao paciente oncológico.

Os cuidados de enfermagem ao paciente com câncer devem ser individualizados principalmente no que tange à idade, pois cada fase da vida apresenta transformações fisiológicas e psíquicas, além de como a visão da morte é encarada. O paciente idoso, por exemplo, está fragilizado pelo natural processo do envelhecimento e com uma perspectiva de sobrevida reduzida; por isso diante de um diagnóstico de uma doença neoplásica maligna, a sua perspectiva se torna bem reduzida e ocorre um grau de sofrimento orgânico considerável. $\mathrm{O}$ enfermeiro deve prover uma maior aproximação com este tipo de paciente, alcançado por meio da comunicação, para identificar suas necessidades e proporcionar melhor qualidade de vida.

O presente estudo teve como objetivo geral o de avaliar as dificuldades e suas respectivas causas na percepção do enfermeiro, ao prestar assistência ao paciente idoso portador de patologia oncológica; e, como objetivo específico, identificar os incidentes críticos (situação, comportamento e consequência) relatados pelos profissionais que prestam assistência de enfermagem ao idoso com doença oncológica. Acredita-se que o estudo poderá contribuir para a reflexão do papel comunicacional do profissional em sua relação com pessoa idosa, portadora de doença oncológica.

\section{MÉTODOS}

Trata-se de um estudo descritivo, a partir do emprego da técnica de incidente crítico junto aos sujeitos. A técnica do incidente crítico $^{(8)}$, é um método indireto de análise do trabaIho e que permite o registro de comportamentos específicos, favorecendo observações e avaliações sistematizadas. Para um incidente ser definido como crítico, ele deve ter uma finalidade ou intenção que esteja clara ao observador e com consequências definidas, a fim de possuir um efeito indubitável.

No Brasil, a técnica foi introduzida pela primeira vez em um estudo para definir critérios para a seleção e avaliação do pessoal $^{(9)}$. No âmbito da Enfermagem brasileira, diversos autores obtiveram sucesso no uso da referida técnica, a exemplo de um estudo que se utilizou da técnica para buscar subsídios para a elaboração de programas de treinamento para os auxiliares de enfermagem ${ }^{(10)}$; de outro, que buscou identificar incidentes críticos da passagem de plantão ${ }^{(11)}$; ou, ainda, a fim de identificar como as mulheres em tratamento de câncer de colo uterino com cesiomoldagem enfrentam esse tratamento ${ }^{(12)}$; ou no estudo que utilizou da mesma técnica para avaliar a percepção do paciente quanto a ter um cateter de longa permanência $^{(13)}$.

A literatura considera a técnica de incidente crítico muito útil para a Enfermagem, ao elucidar comportamentos relativos à $\operatorname{prática}^{(14)}$.

O estudo foi realizado em um hospital geral universitário, localizado no interior do estado de São Paulo, que desenvolve atividades voltadas ao ensino, assistência e pesquisa, desde 1956. É uma entidade integrada ao Sistema Único de Saúde (SUS), prestando assistência de nível terciário. 
A amostra foi composta por enfermeiros que trabalham em Unidades que prestam assistência oncológica às pessoas com idade superior a 60 anos. Foram convidados todos os enfermeiros $(n=56)$ das clínicas médicas, cirúrgicas, central de quimioterapia e áreas de oncologia, que atuam nas áreas de oncologia há mais de um ano. Vale ressaltar que o posicionamento ético da pesquisadora, com relação ao desenvolvimento da pesquisa foi norteado a partir das diretrizes e normas regulamentadoras de pesquisa envolvendo seres humanos, estabelecidas na Resolução no 196/96 do Conselho Nacional de Saúde, em vigor no país. Desse modo, para participar do estudo os enfermeiros manifestaram aquiescência formal por meio do Termo de Consentimento Livre e Esclarecido - TCLE. Dentre os enfermeiros convidados, 30 (53,57\%) participaram da entrevista, sendo que os dados de cinco (8,92\%) foram descartados, por não conterem as informações necessárias à análise proposta no instrumento. Cabe lembrar que os demais $26(46,42 \%)$ se recusaram a participar ou referiram não terem prestado assistência a pacientes idosos, oncológicos e em estado terminal recentemente.

O instrumento utilizado foi composto de duas partes sendo uma para identificação do sujeito com dados de sexo, idade, tempo de formação e tempo de atuação com pacientes idosos em oncologia. A segunda parte, que tratava dos comportamentos e sentimentos sobre Incidente Crítico, foi composta de três questões sobre ocorrências positivas ou negativas (incidente crítico) que envolvem a assistência de portadores de câncer em estado terminal.

Inicialmente foi dada a seguinte orientação ao sujeito: "Pense em uma situação em que você prestou assistência em enfermagem à pessoa idosa portadora de câncer, em estado terminal, sem perspectiva terapêutica, na qual ela tenha comentado sobre o futuro; descreva a situação que ocorreu junto ao paciente, descreva os comportamentos envolvidos nesta situação e mencione os seus sentimentos na situação ocorrida".

Os dados obtidos foram categorizados quanto às características dos sujeitos (idade, sexo, tempo de formado e tempo de atuação em assistência a idosos portadores de doenças oncológicas), bem como aos elementos do incidente crítico (situação, comportamento e emoção).

As respostas foram analisadas por meio da análise de conteúdo de Bardin, para os dados qualitativos, e através de estatísticas simples, para os dados quantitativos, tendo seus resultados quantitativos expostos em porcentagens e os qualitativos em discursos, tendo a pesquisadora feito uma leitura individualizada dos questionários, com a finalidade de se familiarizar, buscando ter uma visão geral de cada um. Após esta etapa, realizou leituras sucessivas e minuciosas de cada questionário, objetivando uma melhor compreensão e atentando para os pontos de convergência que apareciam nestes, dentro do tema estudado, procedendo à identificação dos temas relevantes.

Antecedendo a execução da pesquisa, o projeto foi submetido e aprovado pelo Comitê de Ética em Pesquisa do HC FMRP- USP, observando-se os preceitos estabelecidos pela Resolução no 196/96 do CNS, que normatiza a realização de estudos com seres humanos, de acordo com o Processo
HCRP n 1124/2009. Somente após parecer favorável do Comitê, a pesquisadora dirigiu-se às unidades de clínicas médicas, cirúrgicas, central de quimioterapia e áreas de oncologia, para coleta dos dados da pesquisa.

\section{RESULTADOS}

Os enfermeiros que participaram da pesquisa, pertenciam, em sua maioria, aos setores de Clinica Cirúrgica e Oncologia, apresentaram idade na faixa entre de 23 a 50 anos ( média de 34,6 e desvio padrão de 15,86), tempo de formação de 1,7 a 25 anos (média de 9,36 e desvio padrão de 5,97), e tempo de atuação em idosos em oncologia de 0,25 a 17 anos (média de 5,69 e desvio padrão de 4,04 ). Com relação ao sexo $92 \%$ eram mulheres.

Foram identificados 25 incidentes críticos, que apresentaram diferentes temas: 17 relatos referiram a visita de rotina, um a situação de administração de quimioterapia, um sobre a troca de traqueostomia, dois sobre solicitação de internação, dois relativos a troca de curativo e dois sobre a assistência domiciliar.

Quantos aos comportamentos relatados pelos enfermeiros, os de maior ocorrência foram: estabelecer vínculo, transmitir confiança e credibilidade, procurar fazer o melhor ao paciente, administrar medicamentos prescritos, autorizar visita de familiares, posicionar o paciente da melhor maneira possível, conversar sobre assuntos não relacionados à hospitalização, orientar a paciente, oferecer apoio, manter-se ao seu lado, ouvir o paciente, dedicar-se até o final, animar o paciente, afastar-se, transmitir sentimentos positivos, envolvendo intervenções tanto de caráter técnico e prático quanto de caráter humano.

Em relação à consequência, nove (36\%) foram relatos que representam incidentes críticos negativos e três (12\%) incidentes críticos positivos, sendo que outros 13 (52\%) geraram sentimentos tanto positivos quanto negativos. Nos 25 incidentes críticos foram referidos 57 sentimentos, sendo registrados $22(38,6 \%)$ sentimentos positivos e $35(61,4 \%)$ sentimentos negativos.

Ao se verificar o objeto do sentimento identificamos três diferentes grupos: sentimentos relacionados ao próprio enfermeiro $(57 \%)$ e sentimentos relacionados ao paciente durante a assistência $(36,84)$ e após a assistência $(7 \%)$.

Sentimentos relacionados aos enfermeiros

Quanto aos sentimentos relacionados aos enfermeiros, 12 apontavam sentimentos positivos e 21 sentimentos negativos.

Encontramos os seguintes sentimentos positivos: sensação de dever cumprido, estímulo a valorização da vida, felicidade, satisfação, sentimento de utilidade, reconhecimento do papel da enfermagem com pacientes oncológicos, fortalecimento profissional e emoção positiva pela importância da sua presença. Nesta categoria, apresentou maior frequência o sentimento de dever cumprido (25\%), por terem prestado a assistência com dedicação, preocupação em proporcionar conforto físico e emocional e dignidade.

Os sentimentos de estímulo à valorização da vida e o de felicidade apresentaram frequência de 16,6\% cada. A consequência relatada como felicidade, em todos os relatos, está associada ao fato de o enfermeiro ter realizado uma ação que 
ajudasse o paciente, como autorizar a visita de familiares e trazer sentimentos positivos ao paciente.

Em relação aos sentimentos negativos, encontramos: impotência, angústia, limitações frente às características dos pacientes oncológicos, frustração, desmotivação pelo prognóstico, despreparo pela falta de formação e preparação na graduação para este tipo de atendimento, apreensão e sofrimento. O sentimento de impotência apresentou maior frequência (40\%). Houve relatos, em que, além de impotência, emergiram sentimentos negativos relacionados diretamente com a assistência de enfermagem, caracterizando a dificuldade em utilizar-se de habilidades da enfermagem frente à situação. Foram, ainda, referidas a desmotivação pelo prognóstico do paciente e a falta de formação para esse tipo de atendimento.

Em relação ao despreparo para prestar assistência aos pacientes terminais, o enfermeiro afirma, no relato a seguir, que

[...] não há formação e preparo na graduação para este tipo de atendimento e não há treinamento por parte da instituição hospitalar para preparo dos profissionais [...].

Os sentimentos de angústia e de limitações frente às características dos pacientesoncológicos $(15,7 \%)$ foram citados como exemplo de sentimentos negativos.

\section{Sentimentos relacionados ao paciente durante a assistência}

Quanto aos sentimentos relacionados aos pacientes durante a assistência, foram identificados sete sentimentos positivos e 14 sentimentos negativos.

Foram apontados os seguintes sentimentos positivos: compaixão $(42,8 \%)$, solidariedade $(14,3 \%)$, conforto $(14,3 \%)$, empatia $(14,3 \%)$ e carinho $(14,3 \%)$. Das três citações sobre compaixão, em 2, os enfermeiros se preocuparam em proporcionar uma morte mais digna para o paciente.

Em relação aos sentimentos negativos, foram identificados: tristeza $(57,1 \%)$, sofrimento, dó, dor. Os demais, sofrimento, dó e dor, correspondem a 14,3\%. Em todas as citações, o sentimento de sofrimento estava relacionado também à família do paciente.

Os sentimentos de tristeza, nos relatos, estão relacionados à condição em que o paciente se encontra, ou seja, ao momento em que o enfermeiro conclui que o processo morte-morrer será inevitável para o paciente. Em alguns relatos, o sentimento de impotência também foi referido, juntamente com o sentimento de tristeza. A fim de se distanciar de seus próprios sentimentos negativos frente à situação, os enfermeiros relataram os seguintes comportamentos durante a assistência:

[...] Para conseguir cuidar deste cliente procurei esquecer seu prognóstico e só lembrei quando ele realmente morreu[...]

O distanciamento não foi somente emocional, mas físico como o próprio afastamento do paciente, após o conhecimento do estágio de sua doença.

[...] Afastamento... não conseguia chegar perto da paciente e ver o desespero dela e um pedido de ajuda pelo olhar [...]
No relato a seguir, houve apenas comportamentos focalizados no caráter técnico e prático do cuidado de enfermagem.

[...] Tratei de reconfortá-lo, isto é, posicioná-lo da melhor maneira possível e ver a possibilidade de alguma medicação para este quadro de ansiedade [...]

Nestes recortes, nota-se que houve distanciamento, negação dos sentimentos e cuidados focalizados no caráter técnico e prático, por parte dos enfermeiros, foram encontrados somente sentimentos negativos, ou seja, a atitude dos enfermeiros frente à situação não gerou nenhum tipo de sentimento positivo.

\section{Sentimentos relacionados ao paciente após a assistência}

Quanto aos sentimentos relacionados aos pacientes após a assistência, foram identificados sentimentos positivos de saudades, carinho e respeito ao lembrar-se do paciente e apenas um sentimento negativo, de tristeza pela perda de uma amizade. Os enfermeiros que relataram tais sentimentos estabeleceram fortes vínculos com o paciente, procurando transmitir confiança e credibilidade, estabelecendo uma relação terapêutica.

[...] Apesar da dor, especialmente por perceber a dor vivenciada pelo próximo, há um sentimento de satisfação de saber que foi feito o melhor e que tive contribuição em deixar aqueles momentos finais em algo melhor, digo, tentando melhorar o conforto e segurar a mão, demonstrar carinho e, às vezes, é melhor que um remédio prescrito [...]

\section{A vivência do profissional e os sentimentos emanados}

Embora a amostra seja pequena, algumas associações entre o tipo de sentimento e a vivencia profissional emergiram. Em relação aos sentimentos negativos manifestados pelo profissional, durante a assistência, o de impotência foi apresentado predominantemente por enfermeiros com idades inferiores a 30 anos. Já o sentimento de compaixão apresentado pelos enfermeiros foi citado, em sua maioria, por enfermeiros mais velhos e com um maior tempo de formação, com idades maiores do que 30 anos, tempo de formação maior do que cinco anos. O sentimento de tristeza foi manifestado de forma variada, independente da idade do profissional.

Por outro lado, os enfermeiros que apresentaram sentimentos positivos durante a assistência eram mais velhos, com idades maiores de 30 anos e maior tempo de formação, em sua maioria. Os sentimentos apresentados pelos enfermeiros após a assistência, como os de saudades, respeito, carinho e tristeza, apresentaram idades de 30 anos e tempo de formação maior do que 5 anos e tempo de atuação com idosos em oncologia variados.

\section{DISCUSSÃO}

A fim de que a prestação dos cuidados de enfermagem com pacientes oncológicos seja efetiva, o enfermeiro necessita não só adquirir o conhecimento da patologia em si, mas, 
além disso, a habilidade em lidar com os sentimentos dos outros e com as próprias emoções frente ao doente com ou sem possibilidade de cura.

Estudos que abordem a assistência de enfermagem com pacientes terminais não são frequentes na literatura, principalmente relacionados à comunicação terapêutica, o que justifica a presente pesquisa e sua contribuição para o maior conhecimento das dificuldades que os enfermeiros enfrentam no processo morte-morrer de seus pacientes.

O método utilizado nesta pesquisa permitiu entender os sentimentos e comportamentos dos enfermeiros frente a uma situação na qual os pacientes oncológicos não têm possibilidade de cura; favoreceu também avaliar as dificuldades e as respectivas causas vivenciadas pelos enfermeiros neste contexto assistencial, uma vez que a situação requer o domínio das emoções para que seja possível a comunicação terapêutica entre enfermeiro e paciente.

As situações de assistência com o paciente oncológico, em estado terminal, apresentaram, neste estudo, em maior frequência, incidentes críticos negativos, em especial os sentimentos de impotência e tristeza.

O sentimento de impotência, apresentado pelos enfermeiros, pode ser compreendido pelo fato de os profissionais de enfermagem, diariamente, lutarem pela vida e contra a morte, tomando para si a responsabilidade de salvar, curar ou aliviar, procurando sempre preservar a vida, já que a morte, na maioria das vezes, é vista por estes profissionais como um fracasso ${ }^{(15)}$.

A idade dos profissionais e o tempo de atuação com idosos em oncologia, parece não ter influenciado nos resultados obtidos em relação a expressão do sentimento de tristeza; apesar de os enfermeiros conviverem diariamente com situações de sofrimento diante do processo de morte, necessitam lidar com seus próprios sentimentos e estarem preparados emocionalmente. Os sentimentos de tristeza, apareceram citados, principalmente, durante a assistência de enfermagem relacionados ao acompanhar o sofrimento do paciente e da família frente a evolução para a morte. Conforme frase citada por um autor, "às vezes, o mais difícil não é lidar com a morte e sim acompanhar o paciente vivo que está morrendo"(16).

Em um estudo, os sentimentos despertados ao conversar com um paciente sobre cuidados paliativos foram de impotência e tristeza, relatados pelos profissionais da saúde ${ }^{(17)}$. Em outro estudo foi revelado que os sentimentos que apresentaram maior frequência pelos profissionais de saúde ao atender pacientes oncológicos, em estado terminal, foram de sofrimento, tristeza, angústia e impotência, sentimentos similares aos encontrados nesta pesquisa ${ }^{(18)}$.

Em nossos dados constam relatos nos quais os enfermeiros apresentaram comportamentos de distanciamento, tanto emocional quanto físico, com a finalidade de utilizar de um mecanismo de defesa; este tipo de comportamento é entendido na literatura como o desenvolvimento de uma couraça necessária a proteção contra sentimentos indesejáveis acerca da morte $^{(19)}$. O afastamento também impossibilita ao próprio enfermeiro estabelecer qualquer tipo de vínculo, o que impedirá o início de um relacionamento; para se iniciar um relacionamento terapêutico, é necessário que as pessoas se conheçam e estabeleçam um clima de confiança entre elas ${ }^{(20)}$. Existem autores que destacam que é preciso entender os processos da morte e do morrer para que se torne capaz de auxiliar os pacientes na sua finitude, pois o conhecimento insuficiente deste aspecto poderá levar a um distanciamento do paciente como uma forma de proteção por não saber enfrentar tal situação e uma falha na prestação do cuidado singular/integral tão almejado pela Enfermagem ${ }^{(21)}$.

Segundo autores, em relação à comunicação terapêutica, o enfermeiro deve estar ciente de seus valores, sentimentos e atitudes em relação a este, aprendendo a comunicar - se com ele de modo adequado, já que, aquilo que o profissional sente ou pensa em relação ao indivíduo será transmitidos não verbalmente ${ }^{(22)}$. Isso significa que o enfermeiro não deve negar qualquer sentimento negativo que possa ter em relação a um paciente, dominando, porém, a maneira de expressar seus sentimentos em relação ao mesmo, não o condenando ou censurando.

Porém, os comportamentos voltados ao caráter técnico e prático dos cuidados de enfermagem são aprendidos na graduação, uma vez que a formação dos enfermeiros ainda é centrada no modelo biomédico, sendo o cuidado resumido somente em procedimentos técnicos.

Os comportamentos dos enfermeiros que se preocuparam em proporcionar dignidade aos pacientes, foram ações que englobam o cuidar que, por sua vez, segundo a literatura, são ações que visam a aliviar o sofrimento, manter, promover ou recuperar a dignidade e a totalidade humana ${ }^{(23)}$, geraram os sentimentos de sensação de dever cumprido, estímulo a valorização da vida, felicidade, satisfação, sentimento de utilidade, reconhecimento do papel da enfermagem com pacientes oncológicos, fortalecimento profissional, nos enfermeiros deste estudo, sendo perceptível, pelos mesmos, a melhora da qualidade ao atendimento.

\section{CONSIDERAÇÕES FINAIS}

Os achados deste estudo apontam que os enfermeiros entrevistados, em sua maioria, encontram dificuldades em lidar com os sentimentos negativos, emanados pela situação, interferindo no cuidado de enfermagem prestado aos pacientes. Como citado em alguns relatos, durante a formação profissional houve insuficientes oportunidades para adquirir habilidades de enfermagem para lidar com pacientes terminais, uma vez que, na maioria das vezes, os alunos são preparados para lidarem com a cura e a vida, o que dificulta que os futuros enfermeiros possam prestar um cuidado integral e individualizado aos pacientes em fase terminal. Porém, os enfermeiros que relataram intervenções de caráter humano, demonstraram sentimentos positivos, reconhecendo o quão importante as respectivas ações de enfermagem foram importantes para oferecer uma assistência humana, contribuindo para proporcionar uma morte digna ao paciente. A pesquisa ressalta que este pode ser um bom caminho a ser trabalhado no comportamento dos enfermeiros.

Portanto, profissionais devem ser preparados não somente para possuírem competência técnica, mas para que sejam capazes de lidar com seus próprios sentimentos e atuarem em 
situações de risco de morte, reduzindo o sofrimento pessoal nestas situações.

A fim de que haja uma melhora na qualidade dos serviços prestados por parte dos enfermeiros a estes pacientes, os resultados desta pesquisa sugerem que seja realizada uma capacitação para a assistência paliativa, auxiliando na compreensão de uma morte autenticamente humana, incentivando os enfermeiros a desenvolver habilidades, como a comunicação terapêutica, que auxiliem o paciente a ter uma qualidade de vida digna até o momento de sua morte, viabilizando a humanização do cuidado.

O presente estudo contribui, também, para que os estudantes de Graduação em Enfermagem procurem aprender e trabaIhar habilidades que visem não apenas à assistência voltada ao caráter técnico e prático, mas habilidades de enfermagem que garantam uma assistência integral ao paciente, qualidade de vida e bem-estar, condições indispensáveis para a construção de um modelo assistencial humanizado.

\section{REFERÊNCIAS}

1. Sá ACO. O cuidado do emocional em enfermagem. São Paulo (SP): Robe Editorial; 2001. 114p.

2. Barbosa IA, Silva MJP. Cuidado humanizado em enfermagem: o agir com respeito em um hospital universitário. Rev Bras Enferm, Brasília; 2007;60(5):546-551.

3. Silva MJP. Comunicação tem remédio: a comunicação nas relações interpessoais em saúde. São Paulo: Ed Gente; 1996. $113 \mathrm{p}$

4. Wilkinson S. Factors which influence how nurses communicate with cancer patients. Journal of Advanced Nursing 1991;16(6):677-688.

5. Kmetiuk A., Silva MVZ. Cuidados paliativos de enfermagem em idosos portadores de câncer. Rev. de Enfermagem [Internet] [acesso 29 jul 2008]. Disponível em: < http:// www.uniandrade.edu.br/links/menu3/publicacoes/revista_enfermagem/artigo036.pdf $>$.

6. Silva VCE. O impacto da revelação do diagnóstico de câncer na percepção do paciente [Dissertação]. Ribeirão Preto: Escola de Enfermagem de Ribeirão Preto, Universidade de São Paulo; 2005. Mestrado em Enfermagem Fundamental.

7. Hudak CM., Gallo BM. Efeitos da unidade de terapia intensiva sobre o enfermeiro.In: Hudak C M; Gallo B M. Cuidados intensivos de enfermagem: uma abordagem holística. 6ed. Rio de Janeiro: Guanabara Koogan; 1997. p. 98-109.

8. Flanagan JC. A técnica do incidente crítico. Arq. Bras. Psic. Aplicada, Rio de Janeiro; 1973;25(2): 99-141.

9. DelaColeta JA. A técnica do incidente crítico: aplicações e resultados. Arq. Bras. Psic. Aplicada, Rio de Janeiro;1974;26(2):35-58.

10. Ramos DS. A técnica do incidente crítico: uma aplicação em administração de enfermagem [Dissertação]. Rio de Janeiro: Escola de Enfermagem Anna Nery, Universidade Federal do Rio de Janeiro; 1980. 64f. Mestrado em Enfermagem.

11. Nogueira MS. Incidentes críticos da passagem de plantão [Dissertação]. Ribeirão Preto: Escola de Enfermagem de Ribeirão Preto, Universidade de São Paulo; 1988. 109f. Mestrado em Enfermagem Fundamental.

12. Ravagnani MJC. Vivências de mulheres submetidas à cesio-moldagem [Dissertação]. Ribeirão Preto: Escola de
Enfermagem de Ribeirão Preto, Universidade de São Paulo; 1991. Mestrado em Enfermagem Fundamental.

13. Martins FTM. A percepção do paciente referente a ser portador de um cateter de longa permanência.. Ribeirão Preto. Rev Esc Enferm USP [Internet] 2008 [acesso 12 jun 2009]; 42(3):526-531. Disponível em: < http://www.scielo.br/pdf/reeusp/v42n3/v42n3a15.pdf $>$.

14. Polit DFE, Beck CT, Hungler BB. Fundamentos de pesquisa em enfermagem: métodos, avaliação e utilização. 5ed Porto Alegre: Ed. Artmed; 2004. p.310-356.

15. Magalhães ZR, Santos GF, Caldeira WP. Morte nas instituições de saúde: uma abordagem ética. Enferm Revista 1995;2(4):15-19.

16. Kovács MJ. Os profissionais de saúde e educação e a morte. São Paulo: Casa do psicólogo; 2003.

17. Amaral MXG, Achette D, Barbosa LNF, Bruscatto WL, Kavabata NK. Reações emocionais do médico residente frente ao paciente em cuidados paliativos. Rio de Janeiro. Rev. SBPH [Internet]. 2008 [acesso 11 fev 2010]; 11(1):61-86. Disponível em: < http://scielo.bvs-psi.org.br/scielo.php?pid =S1516$-08582008000100006 \&$ script $=$ sci_arttext $>$.

18. Faria DAP, Maia EMC. Ansiedades e sentimentos de profissionais da enfermagem nas situações de terminalidade em oncologia. Ribeirão Preto. Rev. Latino-Am. Enfermagem [Internet]. 2007. [acesso: 10 fev. 2010]; 15(6): 1131-1137. Disponível em: <http://www.scielo.br/scielo.php?pid $=$ S0104-11692007000600012\&script $=$ sci arttext\&tlng $=\mathrm{pt}>$.

19. Pitta A. Hospital, dor e morte como ofício. São Paulo: Hucitec; 1994.

20. Stefanelli MC. Comunicação com paciente: teoria e ensino. São Paulo: Robe editorial; 1993.

21. Ferreira SS, Neves EP. Ser enfermeira vivenciando o cuidado ao cliente com câncer em tratamento quimioterápico. Rev. Téc. e cient. Enfermagem;2003;1(1): 49-54.

22. Potter AP, Perry AG. Fundamentos de enfermagem: conceitos, processos e prática. Rio de Janeiro: Guanabara Koogan; 2002.

23. Waldow VR. O cuidado na saúde: as relações entre o eu, o outro e os cosmos. Petrópolis: Vozes; 2004. 